\title{
$\nabla$
}

\section{Hypopigmented macules of the limbs in two sisters: report on familial Bier spots}

\author{
Giuseppe Stinco ${ }^{1}$ \\ Pasquale Patrone ${ }^{1}$
}

\author{
Enzo Errichetti ${ }^{1}$
}

DOI: http:/ / dx.doi.org/10.1590/abd1806-4841.20153337

\begin{abstract}
Bier spots are asymptomatic, small, irregular, hypopigmented macules characterized by a normal histological appearance, which are usually found on the arms and legs of young adults. We describe the simultaneous presence of Bier spots in two siblings. This finding is unusual since, to the best of our knowledge, concurrent familial cases have never been reported in the literature.
\end{abstract}

Keywords: Hypopigmentation; Pigmentation skin disorders

\section{INTRODUCTION}

Bier spots are small, irregular, hypopigmented macules characterized by a normal histological appearance, which are usually found on the arms and legs of young adults. ${ }^{1,2}$ They are thought to be a benign, physiologic, vascular anomaly. Since the condition is asymptomatic and usually resolves spontaneously, no specific treatment is required. ${ }^{2}$ We describe the first instance of simultaneous presence of BS in two siblings.

\section{CASE REPORT}

A 35-year-old female and her 38-year-old sister presented to our department with asymptomatic, small, hypopigmented macules distributed symmetrically on the limbs (Figure 1). In both patients, the spots were visible when extremities were placed in a dependent position and disappeared when limbs were raised. Lesions started appearing in the older sister around the age of 25, while in the younger sister, they appeared when she was about 30 . There were no mucosal or nail abnormalities and no associations with acrocyanosis or hyperhidrosis. The women had no history of vascular disease or other relevant patholog- ical conditions. No other family member had had similar lesions. Routine laboratory tests yielded normal results. Tests for cryoglobulin, antiphospholipid antibody, antinuclear antibody, and proteins $S$ and $C$ were all negative. On the basis of the characteristic clinical manifestations, Bier spots (BS) were diagnosed. The patients did not receive treatment.

\section{DISCUSSION}

BS consists of asymptomatic, small, irregular, hypopigmented macules characterized by a normal histological appearance, which are usually found in individuals aged 20-40 and more frequently in women than men. ${ }^{1,2}$ The skin surrounding the lesions may be normal or mildly erythrocyanotic. BS are typically distributed symmetrically on the limbs and tend to appear with venous congestion when patients stand, while they usually disappear if venous flow is enhanced by the elevation of affected extremities. Cases with trunk involvement and generalized lesions have also been described. ${ }^{1}$ Furthermore, BS have rarely been reported in association with conditions such as scleroderma

\footnotetext{
Received on 21.12.2013

Approved by the Advisory Board and accepted for publication on 09.02.2014

Work performed at the Institute of Dermatology, "Santa Maria della Misericordia" University Hospital, Udine, Italy. Financial Support: None.

Conflict of Interest: None.

University of Udine - Udine, Italy.

(C)2015 by Anais Brasileiros de Dermatologia
} 


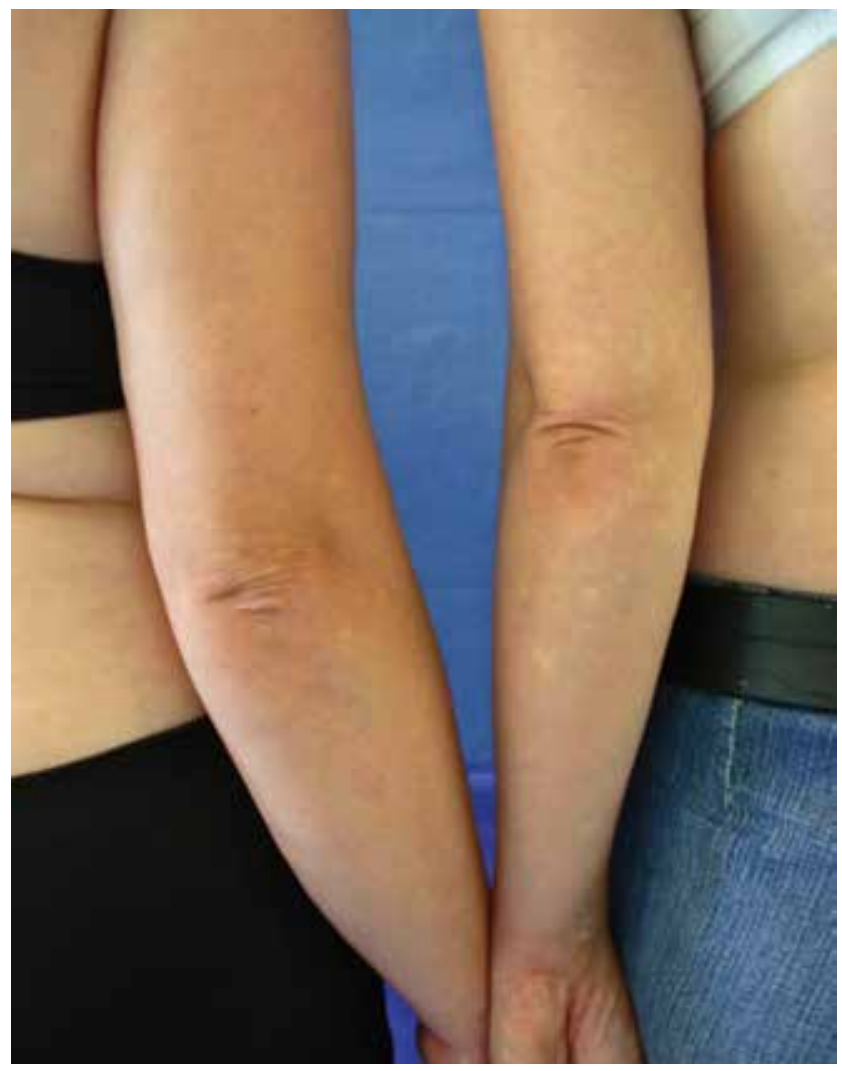

Figure 1:Hypopigmented macules on the older sibling's right arm and on her sister's left arm.

renal crisis, pregnancy, cryoglobulinaemia, coarctation of the aorta, hypoplasia of the aorta, palmar hyperhidrosis, tachycardia, varicosity, lichen planus, alopecia areata and Peutz-Jeghers syndrome. ${ }^{3}$ BS are thought to be an exaggerated but physiological, vasoconstrictive response induced by venous stasis-associated hypoxia or, conversely, by a failure of the venoarteriolar reflex in dermal ascending arterioles in response to venous filling. Other possible pathogenetic mechanisms in- volved in the development of the lesions include rheological abnormalities such as hyperviscosity induced by cryoglobulinaemia. ${ }^{4}$ The main differential diagnoses for BS include disorders either with white macules caused by hypopigmentation (pityriasis versicolor, vitiligo, achromic nevus, postinflammatory hypopigmentation, etc), or those caused by vascular abnormalities (nevus anemicus). ${ }^{1}$ Since the condition usually resolves spontaneously, no specific treatment is required. ${ }^{2}$

In our report we observed the simultaneous presence of BS in two siblings, which is unusual since, to the best of our knowledge, concurrent familial cases have never been reported in the literature.

Although we cannot exclude the possibility that familial occurrence of BS may be casual, we speculate that such an event suggests genetic characteristics may be a factor in the development of the condition, for example a predisposition to an aberrant vasomotor response of the small cutaneous vessels or rheological abnormalities. Importantly, some authors have hypothesized that certain alleles in an autosomal gene locus predispose to skin vasodilatation or vasoconstriction, holding that one of the alleles in particular is responsible for increased vasodilation, and that its homogenous allele accounts for increased vasoconstriction. According to the hypothesis, the functional balance between the two alleles results in a normal vascular tonus; however, some genetic aberrations could lead to an imbalance towards an increased skin vasoconstriction, thus favoring the onset of BS. ${ }^{5}$

In conclusion, this is the first ever report on BS in two siblings; the rarity of familial cases, as well as sporadic forms, is likely due to the fact that the condition is asymptomatic and often goes unnoticed. ${ }^{1} \mathrm{We}$ hope that this report will stimulate further reports and studies, in order to confirm or not the possible role of a genetic component in the genesis of BS.]

\section{REFERENCES}

1. Fan YM, Yang YP, Li W, Li SF. Bier spots: six case reports. J Am Acad Dermatol. 2009;61:e11-2.

2. Liaw FY, Chiang CP. Bier spots. CMAJ. 2013;185:E304

3. Tunca M, Caliskan E, Erbil H, Akar A. Bier spots in two children. Pediatr Dermatol. 2011;28:581-3.

4. Bessis D, Dereure 0, Rivire S, Ravi N, Le Quellec A, Guilhou JJ. Diffuse Bier white spots revealing cryoglobulinaemia. Br J Dermatol. 2002;146:921-2.

5. Tan C, Zhu WY. Unilateral nevoid telangiectasia superimposed on the Bier spots: another demonstration of vascular twin spotting. J Dtsch Dermatol Ges. 2011;9:389-90.

\author{
MAILING ADDRESS: \\ Giuseppe Stinco \\ Institute of Dermatology, "Santa Maria della Misericordia" \\ University Hospital. \\ Piazzale Santa Maria della Misericordia, 15 \\ 33100-Udine, Italy \\ E-mail: giuseppe.stinco@uniud.it
}

How to cite this article: Stinco G, Errichetti E, Patrone P. Hypopigmented macules of the limbs in two sisters: a report of familial Bier spots. An Bras Dermatol. 2015;90(5):738-9. 